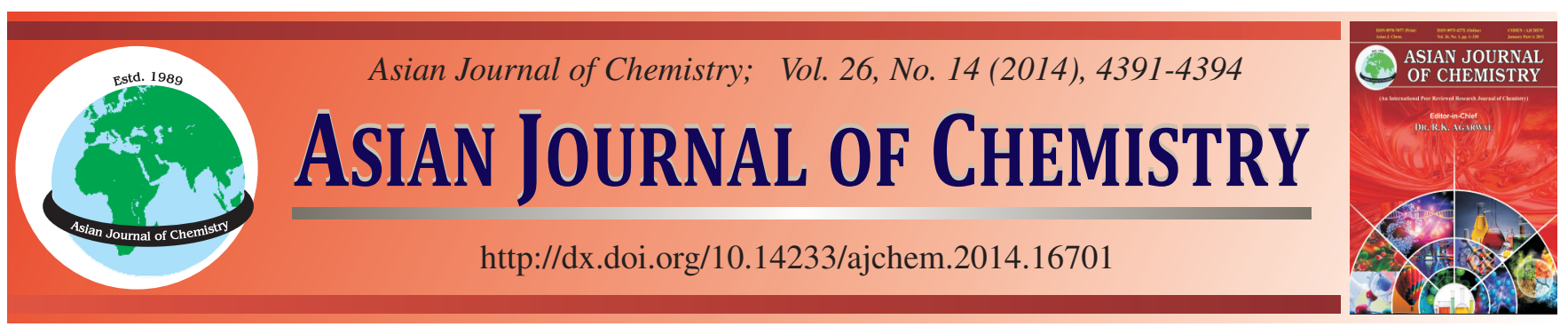

\title{
Extraction and Characterization of Three Polysaccharides from Adhesive Discs of Parthenocissus tricuspidata
}

\author{
L. ZhaNG and W.L. Deng*
}

College of Materials Science and Engineering, South China University of Technology, Guangzhou 510640, P.R. China

*Corresponding author: Tel/Fax: +86 20 22236708; E-mail: wldeng@ scut.edu.cn

In order to reveal the nature of polysaccharides and their contributions to the biological adhesion system of Parthenocissus tricuspidata, three water-soluble polysaccharides named PT1, PT2 and PT3 were extracted and isolated from the adhesive discs. According to the analysis of structure elucidation, they were major composed by $(1 \rightarrow 3)$-linked-Gal $p$ backbone with some branching sites at O- 6 positions and rhamnose, arabinose, xylose and fructose were also present as the side chains. Flocculent structure, worm-like lumps and spherical particles were separately formed by three polysaccharides on the mica surfaces. In addition, branch-like structures and dendritic islands were also observed by atomic force microscopy, which were characterized to be fractal structures by self-similarity.

Keywords: Parthenocissus tricuspidata, Polysaccharide, Adhesive disc.

\section{INTRODUCTION}

Parthenocissus tricuspidata is a tendril-climber liana, belonging to the family of Vitaceae. Following touch stimulation, the tendrils can firmly adhere to different supporting objects by flattening against the support surface, becoming adhesive discs in the tip and subsequently secreting a kind of adhesive compound ${ }^{1-3}$. The unique adhesion ability of $P$. tricuspidata has attracted great attentions from scientists since Darwin's time. Previous studies mainly focus on microstructure observation $^{3-5}$, cytochemical studies ${ }^{1,2,6}$, attachment strength quantification $^{7}$ and bionic fabrication ${ }^{8}$. The adhesive compound secreted from adhesive discs has been considered to be a kind of polysaccharide. However, there are only few reports about the further details of this adhesive compound. Therefore, the adhesion mechanism of $P$. tricuspidata has not been completely confirmed and the further studies on the polysaccharides secreted from adhesive discs are desirable and necessary.

We have communicated a kind of polysaccharide (PT2) from adhesive discs of $P$. tricuspidata before ${ }^{9}$. Here we reported a complete study on the extraction, isolation and characterization of three water-soluble polysaccharides (PT1, PT2 and PT3) from adhesive discs. The chemical compositions and glycosidic linkages were identified by a series of chemical and instrumental methods. In addition, the morphological structures of polysaccharides were investigated by atomic force microscopy (AFM).

\section{EXPERIMENTAL}

UV absorption spectra were measured by an UNICAM UV-500 spectrophotometer. The IR spectra were recorded on a Bruker Vector 33 FTIR spectrometer with $\mathrm{KBr}$ pellet. GC and GC-MS were carried out using an Aglient 6890N instrument equipped with OV-1701 (acid hydrolysis), HP-5 (Smith degradation) and DB-225 (methylation) capillary column.

Extraction and purification of polysaccharides: The adhesive discs of $P$. tricuspidata were collected from Guangzhou, Guangdong Province of mainland China. After pretreatment with $\mathrm{EtOH}$ to remove lipid and pigment, the dried residues were extracted three times with distilled water at $80^{\circ} \mathrm{C}$ for $3 \mathrm{~h}$. The combined aqueous extracts were filtered, concentrated, precipitated with three volumes of $95 \% \mathrm{EtOH}$. The precipitates were collected by centrifugation, washed with ethanol, acetone and ether and dried to obtain a crude polysaccharide. After protein removal, it was dissolved in distilled water and $95 \%$ $\mathrm{EtOH}$ was gradually added to precipitate three fractions. Then, they were purified on a Sephadex G-100 column, eluting with $0.1 \mathrm{M} \mathrm{NaCl}$ at a flow rate of $0.5 \mathrm{~mL} / \mathrm{min}$. The appropriate fractions were combined, dialyzed and dried to give PT1, PT2 and PT3.

Estimation of molecular weight: The average molecular weight was measured on a Waters HPLC apparatus equipped with a Waters 2414 refractive index detector and three columns in series (TSK-GEL Guard Column (PWXL 6.0*40), TSK- 
GEL 4000K pwxL 7.8*300, TSK-GEL 2500 K pwxL 7.8*300). The columns were eluted with $0.2 \mathrm{M} \mathrm{KH}_{2} \mathrm{PO}_{4}$ at a rate of 0.6 $\mathrm{mL} / \mathrm{min}$.

Monosaccharide analysis: The polysaccharide was hydrolyzed with $2 \mathrm{M}$ TFA at $120{ }^{\circ} \mathrm{C}$ for $6 \mathrm{~h}$, reduced with $\mathrm{NaBH}_{4}$ for $3 \mathrm{~h}$ and then acetylated with $\mathrm{Ac}_{2} \mathrm{O}$-pyridine $(1 / 1, \mathrm{v} / \mathrm{v}, 2 \mathrm{~mL})$ at $100{ }^{\circ} \mathrm{C}$ for $1 \mathrm{~h}$. The resulting alditol acetates were subjected to $\mathrm{GC}$ analysis. The temperature program was from 190 to $250{ }^{\circ} \mathrm{C}$ at a healing rate of $5{ }^{\circ} \mathrm{C} / \mathrm{min}$.

Periodate oxidation and Smith degradation: The polysaccharide was oxidized with $0.03 \mathrm{M} \mathrm{NaIO}_{4}$ in the dark at $4{ }^{\circ} \mathrm{C}$. The excess of periodate was consumed with ethylene glycol. The products were reduced with $\mathrm{NaBH}_{4}$, neutralized with acetic acid, dialyzed and then dried. The obtained polyalcohol was hydrolyzed with $2 \mathrm{M}$ TFA at $100{ }^{\circ} \mathrm{C}$ for $6 \mathrm{~h}$, silylated with pyridine-HMDS-TMCS and analyzed by GC. The temperature program was from 85 to $220{ }^{\circ} \mathrm{C}$ at a healing rate of $15^{\circ} \mathrm{C} / \mathrm{min}$.

Methylation analysis: The polysaccharide was methylated according to the method of Ciucanu and Kerek ${ }^{10}$. After hydrolysis with 2 M TFA for $6 \mathrm{~h}$, the methylated polysaccharide was reduced with $\mathrm{NaBH}_{4}$, converted into methylated alditol acetates and analyzed by GC-MS. The temperature program was from 50 to $215^{\circ} \mathrm{C}$ at a healing rate of $40{ }^{\circ} \mathrm{C} / \mathrm{min}$.

Atomic force microscopy: The polysaccharide was dissolved in deionized water to make a $1 \mathrm{mg} / \mathrm{mL}$ solution. A $2 \mu \mathrm{L}$ drop was deposited onto freshly cleaved mica surface and dried in air. AFM images were observed on a Nanoscope Multi-SPM IIIa (Digital Instruments, Santa Barbara, USA) in tapping mode.

\section{RESULTS AND DISCUSSION}

Three water-soluble polysaccharides (PT1, PT2 and PT3) were obtained from adhesive discs of $P$. tricuspidata through pretreatment, extraction with hot water, precipitation by $95 \%$ ethanol, deproteinization and purification on a Sephadex G100 column with a single homogeneous peak (Fig. 1). The homogeneity of polysaccharide was determined by HPGPC and the weight-average molecular weights of three polysaccharides were 24.7, 204.0 and $10.5 \mathrm{kDa}$ according to the calibration curve, respectively. No absorption at $280 \mathrm{~nm}$ and $260 \mathrm{~nm}$ in the UV absorption spectra indicated the absence of protein and nucleic acid.

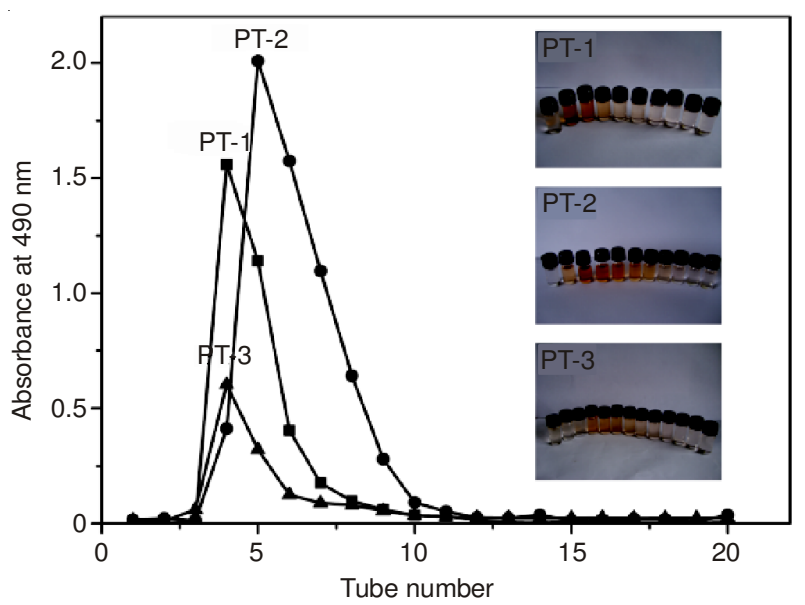

Fig. 1. Elution curves of three polysaccharides on Sephadex G-100 column
The composition of sugars was determined by acid hydrolysis and GC analysis. As shown in Fig. 2, PT1 was composed of rhamnose $(14.3 \%)$, arabinose $(21.7 \%)$, fructose $(30.9 \%)$ and galactose $(33.0 \%)$. PT2 consisted of arabinose (19.6\%), fructose $(44.2 \%)$ and galactose $(36.1 \%)$. Besides rhamnose $(16.1 \%)$, arabinose $(17.0 \%)$, fructose $(24.0 \%)$ and galactose $(30.3 \%)$, PT3 also contained xylose $(12.5 \%)$. These results indicated that galactose and fructose were the major compositions in three polysaccharides.

The IR spectra of PT1, PT2 and PT3 (Fig. 3) showed that the characteristic absorptions of polysaccharide around 3400 and $2950 \mathrm{~cm}^{-1}$ were due to the hydroxyl groups and C-H stretching vibration, respectively ${ }^{11}$. The strong bands between 1200 and $1000 \mathrm{~cm}^{-1}$ were dominated by the glycosidic linkage $v(\mathrm{C}-\mathrm{O}-\mathrm{H})$ and $v(\mathrm{C}-\mathrm{O}-\mathrm{C})$ contributions ${ }^{12,13}$. The weak band at $889 \mathrm{~cm}^{-1}$ existed in the IR spectra of PT1 and PT3 was ascribed to the $\beta$-type glycosidic linkage. For PT2, it contained both $\beta$ - and $\alpha$-type glycosidic linkages according to the absorption bands at 891 and $838 \mathrm{~cm}^{-114}$.

In order to determine the type of glycosidic linkage, three polysaccharides were completely oxidized with $0.03 \mathrm{M} \mathrm{NaIO}_{4}$ in the dark for $72 \mathrm{~h}$. The results showed that $0.83,1.18$ and $0.70 \mathrm{~mol}$ of $\mathrm{NaIO}_{4}$ were consumed and $0.13,0.25$ and $0.12 \mathrm{~mol}$ of formic acid were produced by PT1, PT2 and PT3, respectively. The consumption of periodic acid for each polysaccharide was much more than the production of formic acid, indicating that three polysaccharides contained not only 1,6-linked glycosidic linkage but also 1,2, 1,4 or 1,5-linked glycosidic linkages. After Smith degradation, glycerol and galactose were found by GC, indicating that the C-4 hydroxy group of the glycosyl residues was free. Therefore, PT1, PT2 and PT3 mainly contained 1,2-, 1,5- and 1,6-linked glycosidic linkages, as well as 1,3-linked and/or 1,3,6-linked galactosyl residues.

To further confirm the position of glycosidic linkage, three polysaccharides were completely methylated three times until there were no hydroxy absorption bands in the range of 3600$3000 \mathrm{~cm}^{-1}$ in the IR spectra, followed by GC-MS analysis and the results were given in Table-1. Each of them was a highly branched polysaccharide, containing the non-reducing ends of rhamnose, arabinose or xylose. The arabinosyl units were also substituted at $\mathrm{O}-5$ according to $2,3-\mathrm{Me}_{2}$-Ara derivatives.

\begin{tabular}{|c|c|c|c|}
\hline \multirow{3}{*}{ Alditol acetate } & $\begin{array}{l}\text { TABI } \\
\text { ATION AN } \\
\text { IARIDES FR }\end{array}$ & $\begin{array}{l}\text { SIS OF THF } \\
\text { ADHESIVE }\end{array}$ & \\
\hline & \multicolumn{3}{|c|}{ Type of linkage } \\
\hline & PT1 & PT2 & PT3 \\
\hline $2,3,4-\mathrm{Me}_{3}-\mathrm{Rha}^{\mathrm{a}}$ & 1-Rhap & $\mathrm{nd}^{\mathrm{b}}$ & 1-Rhap \\
\hline 2,3-Me $-\mathrm{Mra}_{2}$ & 1,5-Araf & 1,5-Araf & 1,5-Ara $f$ \\
\hline 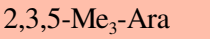 & 1-Araf & 1-Ara $f$ & 1-Araf \\
\hline 2,3,4-Me $-\mathrm{Xly}$ & nd & nd & 1-Xlyp \\
\hline 3,4-Me $\mathrm{Me}_{2}-\mathrm{Man}$ & 1,2,6-Fruf & nd & 1,2,6-Fruf \\
\hline \multicolumn{4}{|l|}{ 3,4-Me $-\mathrm{Glu}$} \\
\hline 3,4,6- $\mathrm{Me}_{3}-\mathrm{Man}$ & nd & 1,2-Fruf & nd \\
\hline \multicolumn{4}{|l|}{ 3,4,6-Me $-\mathrm{Mlu}$} \\
\hline 1,3,4,6- $\mathrm{Me}_{4}-\mathrm{Man}$ & 2-Fruf & 2-Fruf & 2-Fruf \\
\hline \multicolumn{4}{|l|}{$1,3,4,6-\mathrm{Me}_{4}-\mathrm{Glu}$} \\
\hline $2,4-\mathrm{Me}_{2}-\mathrm{Gal}$ & 1,3,6-Galp & $1,3,6-\mathrm{Gal} p$ & $1,3,6-\mathrm{Gal} p$ \\
\hline $2,4,6-\mathrm{Me}_{3}-\mathrm{Gal}$ & 1,3-Galp & $1,3-\mathrm{Gal} p$ & 1,3-Galp \\
\hline
\end{tabular}



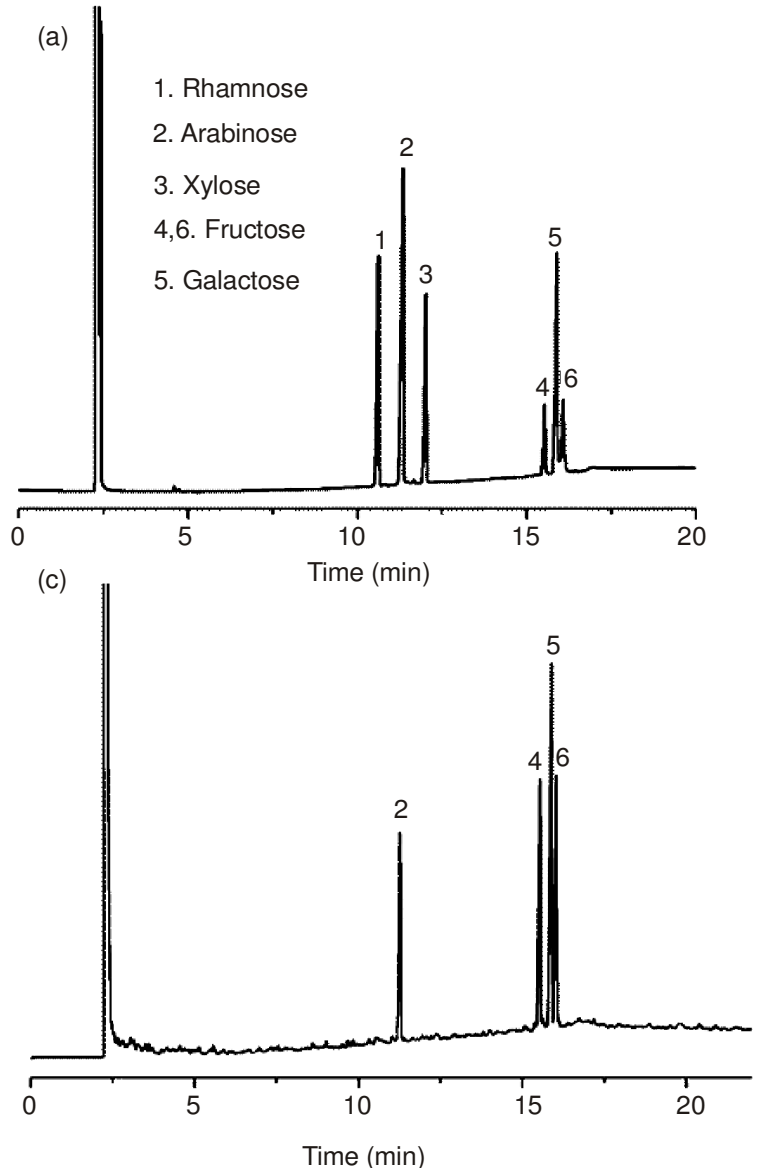
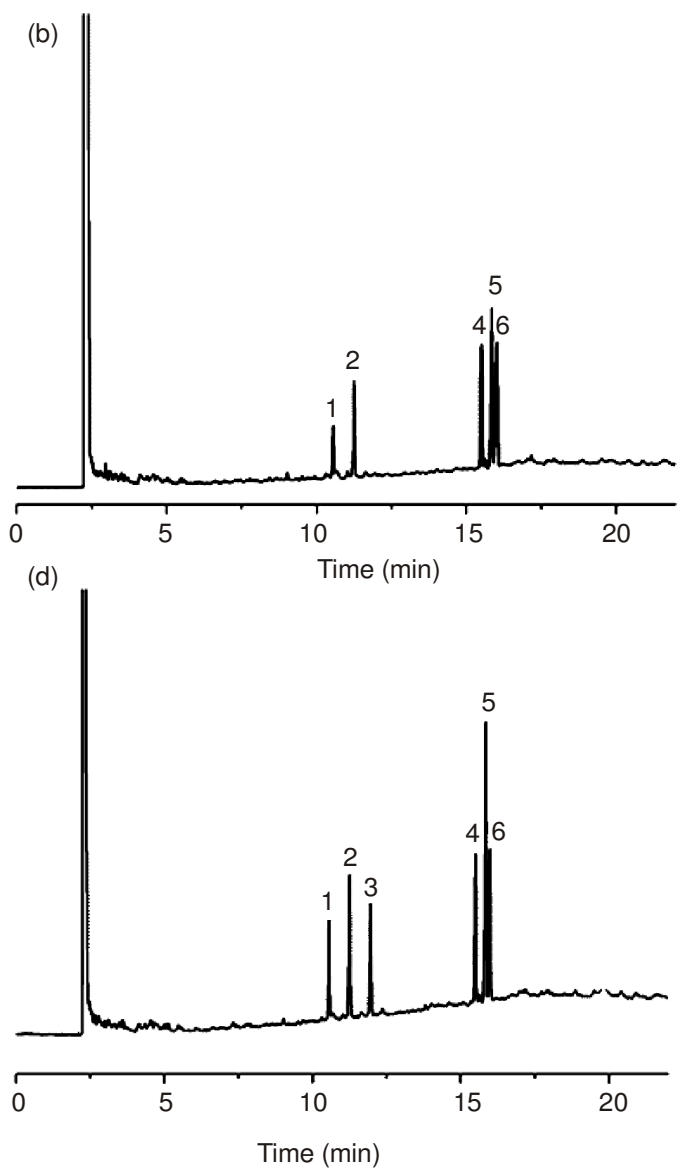

Fig. 2. Gas chromatogram of (a) standard monosaccharides and acid hydrolyzed products of (b) PT1, (c) PT2, (d) PT3. A fructose produced mannitol acetate (peak 4) and glucitol acetate (peak 6) after reduction and acetylation

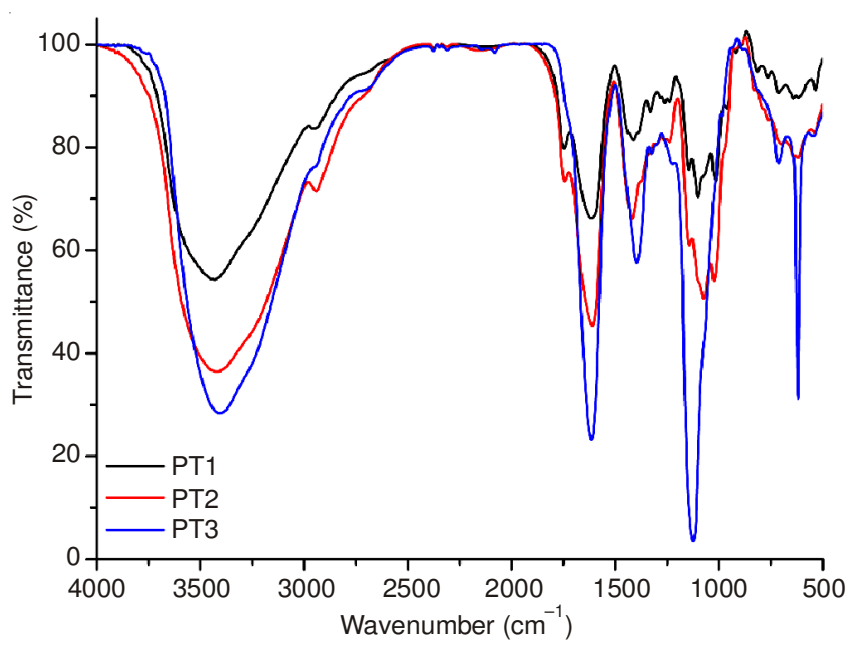

Fig. 3. IR spectra of three polysaccharides extracted from adhesive discs

Generally, a fructosyl residue produced two peaks (mannitol acetate and glucitol acetate) and sum of them represented a certain type of fructosyl residue. Thus, three pairs of peaks,

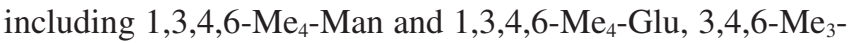

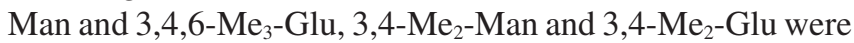
respectively represented $(2 \rightarrow)$-linked, $(1 \rightarrow 2)$-linked and $(1,2$ $\rightarrow 6$ )-linked fructosyl residues. The presence of 2, 4- $\mathrm{Me}_{2}-\mathrm{Gal}$ and 2,4,6- $\mathrm{Me}_{3}-\mathrm{Gal}$ derivatives indicated that the galactose residues were $(1,3 \rightarrow 6)$-linked and $(1 \rightarrow 3)$-linked Galp. These results suggested that PT1, PT2 and PT3 had a $(1 \rightarrow 3)$ -
linked-Galp backbone with some branching sites at O-6 positions. The conclusions of methylation were also in well agreement with the analysis of periodate oxidation and Smith degradation.

Atomic force microscopy works by a very sharp tip over a sample surface, is an excellent tool to characterize biopolymers and it has been extensively used to study the morphological structure of polysaccharide with high resolution under ambient conditions. As shown in Fig. 4a, a type of heterogeneous flocculent structure which consisted of a large number of aggregates was formed on mica surface by PT1 under the concentration of $1 \mathrm{mg} / \mathrm{mL}$. The side chains of PT1 and higher concentration of polysaccharide solution were responsible for the formation of this heterogeneous structure. PT2 formed a homogeneous film on the mica surface and in the magnification image, worm-like lumps with the average height of about $7 \mathrm{~nm}$ were observed by AFM (Fig. 4b), indicating that PT2 could absorb water in the aqueous solution ${ }^{15}$. In addition, the wormlike structure was also caused by the random coil of branch chains ${ }^{16}$. Different from PT1 and PT2, the spherical particles with the diameter ranged from 100 to $150 \mathrm{~nm}$ were observed from the AFM image of PT3. Generally, the size of individual molecular chain for polysaccharide was $0.1-1.0 \mathrm{~nm}$. Therefore, the spherical particle formed by PT3 was the aggregation of sugar chains rather than the single molecule of polysaccharide. Moreover, we also observed some branch-like structures (Fig. 5a and 5b) and dendritic islands with irregular shapes 

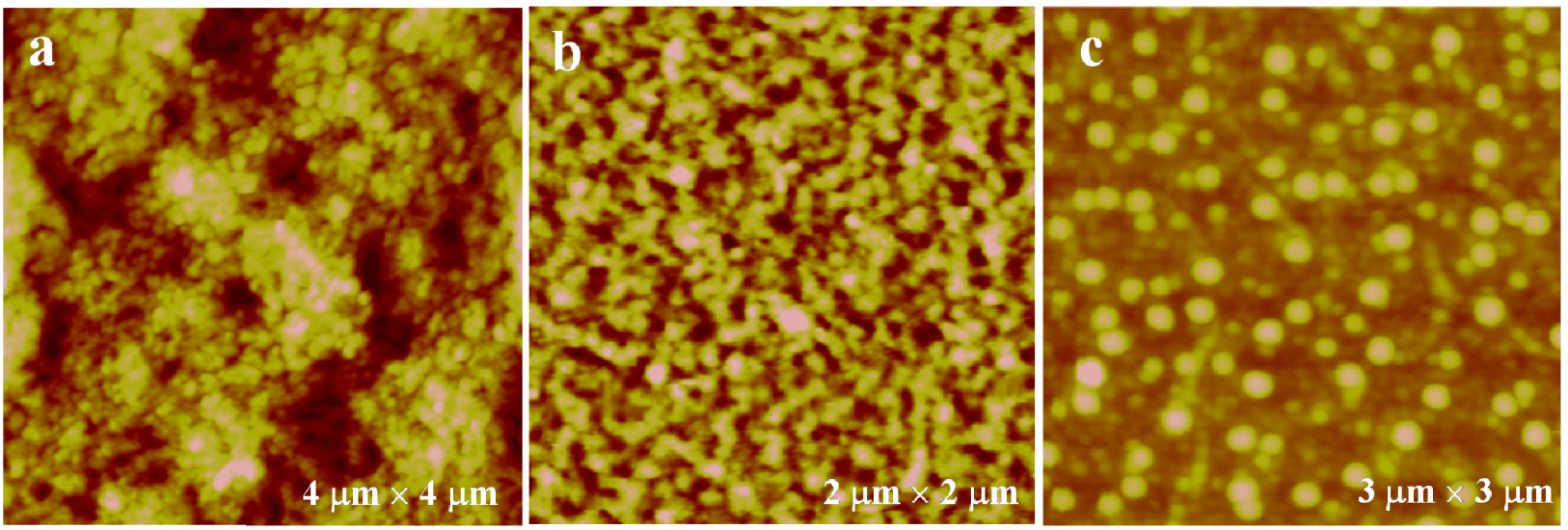

Fig. 4. AFM images of (a) PT1, (b) PT2 and (c) PT3 under the concentrations of $1 \mathrm{mg} / \mathrm{mL}$
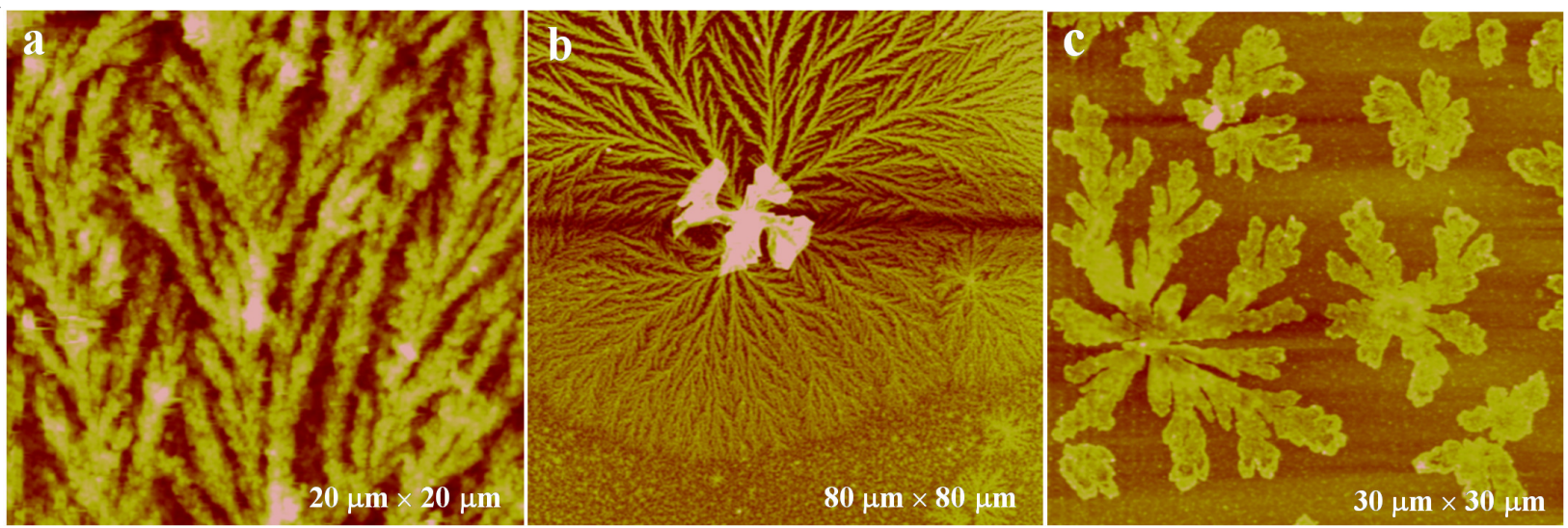

Fig. 5. Fractal structures formed on mica surfaces by (a) PT1, (b) PT2 and (c) PT3 under the concentration of $1 \mathrm{mg} / \mathrm{mL}$

(Fig. 5c) at the edges of three samples, which were the typical fractal structure characterized by self-similarity. The polysaccharide solution could be considered as a non-equilibrium system during drying. Solutions volatilize from the substrate boundary to the center ${ }^{17}$. The nucleation effect when the local concentration reached corresponding critical value led to the formation of these fractal structures on mica surfaces ${ }^{18}$.

\section{Conclusion}

The present study is the comprehensive report on the structure elucidation of three water-soluble polysaccharides from adhesive discs of $P$. tricuspidata. We have designed and fabricated a kind of polystyrene honeycomb-like microstructure based on the adhesive disc. The results of this work provided a considerable access for the further studies on the preparation of advanced composite adhesive material inspired by the biological adhesion system of $P$. tricuspidata.

\section{ACKNOWLEDGEMENTS}

Financial supports from the National Program on Key Basic Research Project (2012CB932900 and 2009CB930604), the National Natural Science Foundation of China (21103053, 91023002, 51373055 and 51073059) and the Cooperation Project in Industry, Education and Research of Guangdong Province and Ministry of Education of China (No. 2011B090400376) are gratefully acknowledged.

\section{REFERENCES}

1. A.G. Endress and W.W. Thomson, Can. J. Bot., 55, 918 (1977).

2. A.J. Bowling and K.C. Vaughn, Protoplasma, 232, 153 (2008).

3. T. He, Z. Li and W. Deng, Arch. Biol. Sci., 63, 393 (2011).

4. C. Darwin, The Movements and Habits of Climbing Plants, John Murray, London (1875).

5. S. Junker, New Phytol., 77, 741 (1976).

6. A.G. Endress and W.W. Thomson, Protoplasma, 88, 315 (1976).

7. T. Steinbrecher, E. Danninger, D. Harder, T. Speck, O. Kraft and R. Schwaiger, Acta Biomater., 6, 1497 (2010).

8. T.X. He, L. Zhang and W.L. Deng, Mater. Chem. Phys., 131, 23 (2011).

9. L. Zhang and W.L. Deng, Asian J. Chem., 25, 874 (2013).

10. I. Ciucanu and F. Kerek, Carbohydr. Res., 131, 209 (1984).

11. C.F. Hu, J.H. Li, D.Y. Yang, Y.J. Pan and H.T. Wan, J. Nat. Prod., 73, 1489 (2010).

12. M.B. Wu, Y.L. Wu, J. Zhou and Y.J. Pan, Food Chem., 113, 1020 (2009).

13. X.H. Chen, Y.H. Liu, X. Bai, L. Wen, J.B. Fang, M. Ye and J.C. Chen, J. Nat. Prod., 72, 1988 (2009).

14. S.A. Barker, E.J. Bourne, M. Stacey and D.H. Whiffen, J. Chem. Soc., 171 (1954)

15. F. Tao, G. Zheng Biao, J. Zheng Yu and Z. Hai Ning, Carbohydr. Polym., 71, 159 (2008).

16. X. Ding, S. Feng, M. Cao, M.T. Li, J. Tang, C.X. Guo, J. Zhang, Q. Sun, Z.R. Yang and J. Zhao, Carbohydr. Polym., 81, 942 (2010).

17. X.H. Duan, C.J. Liu, Y.L. Qiao, Y. Zhou, F.D. Nie, C.H. Pei and J. Chen, J. Cryst. Growth, 351, 56 (2012).

18. Y.H. Shen, A.J. Xie, J.Z. Zhang, F.Z. Cui and H.G. Zhu, Physica B, 363, 61 (2005). 